\title{
THE INTEGRABILITY OF GENERALIZED GARRETT-STANOJEVIĆ SUMS
}

\author{
NIRANJAN SINGH AND K. M. SHARMA
}

(Communicated by Richard R. Goldberg)

\begin{abstract}
In this paper we have defined the generalized Garrett-Stanojevic cosine sums

$$
h_{n}(x)=\sum_{p=0}^{n} S_{p}^{r-1} \Delta^{r} a_{p}
$$

and have proved that under suitable conditions $h_{n} \rightarrow h$ in the $L^{1}$-norm, where $h(x)=a_{0} / 2+\sum_{n=1}^{\infty} a_{n} \cos n x$. If $r=1$, then $h_{n}(x)$ reduces to the modified cosine sums introduced by Rees and Stanojević.
\end{abstract}

1. Introduction. Let

$$
\begin{aligned}
h(x) & =\frac{a_{0}}{2}+\sum_{n=1}^{\infty} a_{n} \cos n x \\
g_{n}(x) & =\frac{1}{2} \sum_{i=0}^{n} \Delta a_{i}+\sum_{i=1}^{n}\left(\sum_{j=i}^{n} \Delta a_{j}\right) \cos i x .
\end{aligned}
$$

In [8] J. W. Garrett and Č. V. Stanojević, using modified cosine sums (1.2) from [6], proved the following result.

THEOREM A. Let $\left\{a_{n}\right\}$ be a null sequence of bounded variation. Then the sequence of modified cosine sums

$$
g_{n}(t)=S_{n(t)}-a_{n+1} D n(t),
$$

where the $S_{n}$ are partial sums of the cosine series (1.1) and $D_{n}$ is Dirichlet kernel, converges in $L^{1}(0, \pi)$-norm to $h$, the pointwise sum of the cosine series, if and only if

(C) for every $\varepsilon>0$ there exists $\partial(\varepsilon)>0$, independent of $n$, such that

$$
\int_{0}^{\partial}\left|\sum_{k=n+1}^{\infty} \Delta a_{k} D_{k}(t)\right| d t<\varepsilon
$$

for every $n$.

This result contains as a special case a number of classical and neoclassical results. In particular, in [8] the following corollary to Theorem A is proved.

Received by the editors July 20, 1987.

1980 Mathematics Subject Classification (1985 Revision). Primary 42A20, 42A32.

Key words and phrases. Cesàro means, $L^{1}$-convergence, generalized cosine sums. 
THEOREM B. Let $\left\{a_{n}\right\}$ be a null sequence of bounded variation satisfying the conditions (C). Then the cosine series is the Fourier series of its sum $h$ and

$$
\left\|S_{n}(h)-h\right\|=o(1), \quad n \rightarrow \infty,
$$

is equivalent to

$$
a_{n} \log n=o(1), \quad n \rightarrow \infty .
$$

In [1] A. N. Kolmogorov introduced quasiconvex sequences and proved his wellknown result:

THEOREM C. If $\left\{a_{n}\right\}$ is a quasiconvex null sequence, then for the convergence of the series (1.1) in the metric space $L^{1}$ it is necessary and sufficient that

$$
\lim _{n \rightarrow \infty} a_{n} \log n=0 .
$$

Applying the partial summation formula to $g_{n}(x)$, we easily see that

$$
g_{n}(x)=\sum_{p=0}^{n} S_{p}^{0}(x) \Delta a_{p},
$$

where $S_{p}^{0}(x)=D_{p}(x)$, which can be interpreted as the partial sum of the series

$$
\frac{1}{2}+\cos x+\cos 2 x+\cdots+\cos n x+\cdots .
$$

Thus in Theorem A, Garrett and Stanojević have studied the $L^{1}$-norm convergence of $\sum_{p=0}^{n} S_{p}^{0}(x) \Delta a_{p}$ to $h(x)$. It is natural to seek ways to prove $L^{1}$-norm convergence of the generalized sums of the form $\sum_{p=0}^{n} S_{p}^{r-1}(x) \Delta^{r} a_{p}$ to $h$, where $r$ is any real number, $r-1 \geq 0, S_{p}^{r-1}(x)$ is the Cesàro sum of order $(r-1)$ of the series (1.4), and $\Delta^{r} a_{p}$ is the difference of order $r$ of $a_{p}$. It is obvious that for $r=1$

$$
\sum_{p=0}^{n} S_{p}^{r-1}(x) \Delta^{r} a_{p}=g_{n}(x) .
$$

Therefore we define

$$
h_{n}(x)=\sum_{p=0}^{n} S_{p}^{r-1}(x) \Delta^{r} a_{p}, \quad(r \geq 1)
$$

and call it the generalized Garrett-Stanojević sum.

The purpose of this paper is to prove the $L^{1}$-norm convergence of $h_{n}$ to $h$ under certain conditions on the coefficients $a_{n}$ and the parameter $r$.

In [2] C. N. Moore generalized quasiconvexity of null sequences in the following way:

$$
\sum_{n=1}^{\infty} n^{k}\left|\Delta^{k+1} a_{n}\right|<\infty, \quad \text { for } k>0,
$$

where the order of differences is fractional; and he proved the corresponding integrability result.

It is well known [4] that if $\left\{a_{n}\right\}$ is a null sequence satisfying the condition (M), then

$$
\sum_{n=1}^{\infty} n^{r}\left|\Delta^{r+1} a_{n}\right|<\infty
$$


for $0 \leq r<k$. In particular it is of bounded variation.

Singh and Sharma [9] have recently obtained a relation between the classes (C) and (M) as follows:

THEOREM D. Let $\left\{a_{n}\right\}$ be a null sequence satisfying condition (M). Then it satisfies condition $(\mathrm{C})$.

In other words, Moore's class (M) is a subclass of the class (C).

This theorem supplies an interesting and nontrivial example to Theorem B.

The proof of Theorem $\mathrm{D}$ essentially depends on the following theorem:

THEOREM E [9]. Let $k>0$ be a real number. If

$$
\begin{aligned}
& \lim _{n \rightarrow \infty} a_{n}=0, \\
& \sum_{n=1}^{\infty} n^{k}\left|\Delta^{k+1} a_{n}\right|<\infty,
\end{aligned}
$$

then $g_{n}(x)$ converges to $h(x)$ in the $L^{1}$-metric.

If we take $k=1$, then this theorem reduces to the following theorem of Garrett and Stanojević [7].

THEOREM F. If $\left\{a_{n}\right\}$ is a null quasiconvex sequence, then $g_{n}(x)$ converges to $h(x)$ in the $L^{1}$-norm.

As a consequence of Theorems B and D, Singh and Sharma [9] have obtained a generalization of Theorem $\mathrm{C}$ in the form of the following theorem.

THEOREM G. Let $k$ be a real number such that $k>0$. If

$$
\begin{aligned}
& \lim _{n \rightarrow \infty} a_{n}=0, \\
& \sum_{n=1}^{\infty} n^{k}\left|\Delta^{k+1} a_{n}\right|<\infty,
\end{aligned}
$$

then for the convergence of the series (1.1) in the metric space $L^{1}$ it is necessary and sufficient that $\lim _{n \rightarrow \infty} a_{n} \log n=0$.

Now we are able to prove the $L^{1}$-norm convergence of the generalized GarrettStanojevic sums $h_{n}$ in the form of the following

THEOREM. Let $k>0$ be a real number. If

$$
\begin{gathered}
\lim _{n \rightarrow \infty} a_{n}=0, \\
\sum_{n=0}^{\infty}(n+1)^{k}\left|\Delta^{k+1} a_{n}\right|<\infty,
\end{gathered}
$$

then for $k \leq r \leq k+1$,

(i) $h_{n}(x)$ converges to $h(x)$ pointwise for $0<\delta \leq x \leq \pi$, and

(ii) $h_{n} \rightarrow h$ in the metric space $L^{1}$.

REMARKS. 1. In our theorem if we take $k=1$ and $r=1$, Theorem $\mathrm{F}$ is obtained as a particular case, since for $r=1, h_{n}(x)=g_{n}(x)$. 
2. Theorem $\mathrm{E}$ can also be deduced as a particular case from our theorem if we take $r=1$ only.

3. The generalized cosine sums $h_{n}(x)$ involve a parameter $r$, and condition (b) of our theorem involves a parameter $k$. Thus we have different criteria for $L^{1}$ convergence of $h_{n}(x)$ for different values of $r$ and $k$.

2. Notations and formulae. Let $\sum a_{n}$ be a given infinite series. For any real number $k$ the Cesàro sums of order $k$ of $\sum a_{n}$ are defined by

$$
S_{n}^{k}\left(a_{p}\right)=S_{n}^{k}=\sum_{p=0}^{n} A_{n-p}^{k} a_{p}=\sum_{p=0}^{n} A_{n-p}^{k-1} S_{p}
$$

where $S_{n}=S_{n}^{0}=a_{0}+a_{1}+\cdots+a_{n}$, and $A_{p}^{k}$ denotes the binomial coefficients.

The Cesàro means $T_{n}^{k}$ of order $k$ of $\sum a_{n}$ will be defined by

$$
T_{n}^{k}=S_{n}^{k} / A_{n}^{k} \text {. }
$$

The following formulae will also be needed.

$$
\begin{gathered}
S_{n}^{k}\left(S_{p}^{r}\right)=S_{n}^{k+r+1}, \\
S_{n}^{k+1}-S_{n-1}^{k+1}=S_{n}^{k}, \quad \sum_{p=0}^{n} A_{n-p}^{\alpha} A_{p}^{\beta}=A_{n}^{\alpha+\beta+1} .
\end{gathered}
$$

We will denote by $S_{n}^{k}(x)$ and $T_{n}^{k}(x)$ the Cesàro sum and Cesàro mean of order $k$ respectively of the series (1.4).

For any positive integer $\alpha$, the differences of order $\alpha$ of a sequence $\left\{a_{n}\right\}$ are defined by the equations

$$
\Delta^{1} a_{n}=a_{n}-a_{n+1}, \quad \Delta^{\alpha} a_{n}=\Delta^{1}\left(\Delta^{\alpha-1} a_{n}\right) .
$$

For these differences, we have the formula

$$
\Delta^{\alpha} a_{n}=\sum_{m=0}^{\alpha} A_{m}^{-\alpha-1} a_{n+m}=\sum_{m=0}^{\infty} A_{m}^{-\alpha-1} a_{n+m} .
$$

If the series (2.5) converges for some $\alpha$ which is not a positive integer, we define

$$
\Delta^{\alpha} a_{n}=\sum_{m=0}^{\infty} A_{m}^{-\alpha-1} a_{n+m} .
$$

The broken differences $\Delta_{n}^{\alpha} a_{p}$ are defined by

$$
\Delta_{n}^{\alpha} a_{p}=\sum_{m=0}^{n-p} A_{m}^{-\alpha-1} a_{p+m} .
$$

By means of the broken differences, the generalized Abel transformation of order $\alpha$ is given by

$$
\sum_{p=0}^{n} a_{p} b_{p}=\sum_{p=0}^{n} S_{p}^{\alpha-1} \Delta_{n}^{\alpha} b_{p}
$$


3. For the proof of our theorem we need the following lemmas:

LEMMA 1 [4]. If $\alpha \geq 0$,

(i) $\varepsilon_{n}=O(1)$, and

(ii) $\sum_{n=0}^{\infty} A_{n}^{\alpha}\left|\Delta^{\alpha+1} \varepsilon_{n}\right|<\infty$,

then $A^{\lambda} \Delta^{\lambda} \varepsilon_{n}$ tends to zero as $n \rightarrow \infty$ for $0 \leq \lambda \leq \alpha$.

LEMMA 2 [5]. Let $r$ be a real number $\geq 0$. If the sequence $\left\{\varepsilon_{n}\right\}$ satisfies the conditions:

(i) $\varepsilon_{n}=O(1)$, and

(ii) $\sum_{n=0}^{\infty} n^{r}\left|\Delta^{r+1} \varepsilon_{n}\right|<\infty$, then

$$
\Delta^{\beta} \varepsilon_{n}=\sum_{m=0}^{\infty} A_{m}^{r-\beta} \Delta^{r+1} \varepsilon_{n+m}, \quad \text { for } \beta>0 .
$$

LEMMA 3 [2]. Let $k$ be a real number $>0$. Then $\int_{0}^{\pi}\left|T_{n}^{k}(x)\right| d x$ remains bounded for all $n$.

LEMMA 4. Let $k>0$. When $0<\delta<1$, then for $0 \leq n<m$, we have

$$
\left|\sum_{p=0}^{n} S_{p}^{k-\delta}(x) A_{m-p}^{\delta-1}\right| \leq C A_{m}^{k}, \quad \text { for } 0<\varepsilon \leq x \leq \pi .
$$

The proof of this lemma is just a slight modification of the proof of Lemma 1 of Andersen [5].

PROOF. To prove this lemma we apply the Abel transformation of order $\delta$ to the broken sum and obtain

$$
\sum_{p=0}^{n} S_{p}^{k-\delta}(x) A_{m-p}^{\delta-1}=\sum_{p=0}^{n} S_{p}^{k}(x) \sum_{\mu=0}^{n-p} A_{\mu}^{-\delta-1} A_{m-p-\mu}^{\delta-1} .
$$

Since $n<m$ and the binomial coefficients $A_{\mu}^{\delta-1}$ are all positive, while the coefficients $A_{\mu}^{-\delta-1}$ are negative for $\mu>0$, we have for $0 \leq p \leq n$

$$
\sum_{\mu=0}^{n-p} A_{\mu}^{-\delta-1} A_{m-p-\mu}^{\delta-1}>\sum_{\mu=0}^{m} A_{\mu}^{-\delta-1} A_{m-p-\mu}^{\delta-1}=A_{m-p}^{-1}=0
$$

and consequently

$$
\left|\sum_{p=0}^{n} S_{p}^{k-\delta}(x) A_{m-p}^{\delta-1}\right| \leq \sum_{p=0}^{n}\left|S_{p}^{k}(x)\right| \sum_{\mu=0}^{n-p} A_{\mu}^{-\delta-1} A_{m-p-\mu}^{\delta-1} .
$$

It is known that $S_{p}^{k}(x)$ is bounded for $0<\varepsilon \leq x \leq \pi$, and so by (3.1), we have

$$
\begin{aligned}
\left|\sum_{p=0}^{n} S_{p}^{k-\delta}(x) A_{m-p}^{\delta-1}\right| & \leq C \sum_{p=0}^{n} A_{p}^{k} \sum_{\mu=0}^{n-p} A_{\mu}^{-\delta-1} A_{m-p-\mu}^{\delta-1} \\
& =C \sum_{p=0}^{n} A_{p}^{k-\delta} A_{m-p}^{\delta-1} \\
& \leq C \sum_{p=0}^{m} A_{m-p}^{\delta-1} A_{p}^{k-\delta} \\
& =C A_{m}^{k},
\end{aligned}
$$


where $C$ is an absolute constant which may be different at different occurrences.

This proves the lemma.

4. Proof of the Theorem. Under conditions (a) and (b) of our theorem it has been proved by $\mathrm{C}$. N. Moore [2] that the series (1.1) converges in the open interval $(0<x \leq \pi)$ to an $L^{1}$-integrable function $h(x)$ and

$$
h(x)=\frac{a_{0}}{2}+\sum_{n=1}^{\infty} a_{n} \cos n x=\sum_{n=0}^{\infty} S_{n}^{k}(x) \Delta^{k+1} a_{n} .
$$

The series on the right-hand side of (4.1) is absolutely and uniformly convergent for $0<\delta \leq x \leq \pi$, so it can be integrated term by term. We prove our theorem in three different cases.

Case (1). Let $r=k+1$. Then

$$
h_{n}(x)=\sum_{p=0}^{n} S_{p}^{k}(x) \Delta^{k+1} a_{p}
$$

So by (4.1) $h_{n}(x) \rightarrow h(x)$ pointwise for $0<\delta \leq x \leq \pi$ and $h(x)$ is Lebesgue integrable.

Now

$$
\begin{aligned}
\int_{0}^{\pi} \mid & h(x)-h_{n}(x)\left|d x=\int_{0}^{\pi}\right| \sum_{p=0}^{\infty} S_{p}^{k}(x) \Delta^{k+1} a_{p}-\sum_{p=0}^{n} S_{p}^{k}(x) \Delta^{k+1} a_{p} \mid d x \\
& =\int_{0}^{\pi}\left|\sum_{p=n+1}^{\infty} S_{p}^{k}(x) \Delta^{k+1} a_{p}\right| d x \\
& \leq \sum_{p=n+1}^{\infty} \int_{0}^{\pi}\left|S_{p}^{k}(x)\right| d x\left|\Delta^{k+1} a_{p}\right| \\
& =\sum_{p=n+1}^{\infty} A_{p}^{k}\left|\Delta^{k+1} a_{p}\right| \int_{0}^{\pi}\left|T_{p}^{k}(x)\right| d x \\
& \leq C \sum_{p=n+1}^{\infty} A_{p}^{k}\left|\Delta^{k+1} a_{p}\right|, \quad \text { by Lemma } 3 \\
& =o(1), \quad \text { by the hypothesis of the theorem. }
\end{aligned}
$$

So we have

$$
\lim _{n \rightarrow \infty} \int_{0}^{\pi}\left|h(x)-h_{n}(x)\right| d x=0
$$

and thus $h_{n} \rightarrow h$ in the $L^{1}$-metric.

Case (2). Let $k<r<k+1$. Then for these values of $r, h_{n}(x)$ can be written as

$$
h_{n}(x)=\sum_{p=0}^{n} S_{p}^{k-\delta}(x) \Delta^{k-\delta+1} a_{p}, \quad 0<\delta<1 .
$$


By Lemma 2 and applying an Abel transformation of the order $-\delta$, we obtain

$$
\begin{aligned}
\sum_{p=0}^{n} S_{p}^{k}(x) \Delta^{k+1} a_{p} & =\sum_{p=0}^{n} S_{p}^{k-\delta}(x) \sum_{\mu=0}^{n-p} A_{\mu}^{\delta-1} \Delta^{k+1} a_{p+\mu} \\
& =\sum_{p=0}^{n} S_{p}^{k-\delta}(x) \Delta^{k-\delta+1} a_{p}-R_{n}(x)
\end{aligned}
$$

where

$$
\begin{aligned}
R_{n}(x)= & \sum_{p=0}^{n} S_{p}^{k-\delta}(x)\left[A_{n+1-p}^{\delta-1} \Delta^{k+1} a_{n+1}+A_{n+2-p}^{\delta-1} \Delta^{k+1} a_{n+2}+\cdots\right] \\
= & \left(\sum_{p=0}^{n} S_{p}^{k-\delta}(x) A_{n+1-p}^{\delta-1}\right) \Delta^{k+1} a_{n+1} \\
& +\left(\sum_{p=0}^{n} S_{p}^{k-\delta}(x) A_{n+2-p}^{\delta-1}\right) \Delta^{k+1} a_{n+2}+\cdots
\end{aligned}
$$

This implies that

$$
\begin{aligned}
h_{n}(x) & =\sum_{p=0}^{n} S_{p}^{k-\delta}(x) \Delta^{k-\delta+1} a_{p} \\
& =\sum_{p=0}^{n} S_{p}^{k}(x) \Delta^{k+1} a_{p}+R_{n}(x) .
\end{aligned}
$$

Now by Lemma 4 , for $0<\delta \leq x \leq \pi$,

$$
\begin{aligned}
\left|R_{n}(x)\right| & \leq C A_{n+1}^{k}\left|\Delta^{k+1} a_{n+1}\right|+C A_{n+2}^{k}\left|\Delta^{k+1} a_{n+2}\right|+\cdots \\
& =o(1), \text { by the hypothesis of the theorem. }
\end{aligned}
$$

Therefore, $h_{n}(x)$ converges to $h(x)$ as $n \rightarrow \infty$ for $0<\delta \leq x \leq \pi$.

Again

$$
\begin{aligned}
\int_{0}^{\pi} & \left|h(x)-h_{n}(x)\right| d x=\int_{0}^{\pi}\left|\sum_{p=n+1}^{\infty} S_{p}^{k}(x) \Delta^{k+1} a_{p}-R_{n}(x)\right| d x \\
& \leq \sum_{p=n+1}^{\infty} \int_{0}^{\pi}\left|T_{p}^{k}(x)\right| d x A_{p}^{k}\left|\Delta^{k+1} a_{p}\right|+\int_{0}^{\pi}\left|R_{n}(x)\right| d x \\
& \leq C \sum_{p=n+1}^{\infty} A_{p}^{k}\left|\Delta^{k+1} a_{p}\right|+\int_{0}^{\pi}\left|R_{n}(x)\right| d x \\
& =o(1)+\int_{0}^{\pi}\left|R_{n}(x)\right| d x
\end{aligned}
$$

by the hypothesis of the theorem and Lemma 3 . 
Now we estimate

$$
\begin{aligned}
\int_{0}^{\pi}\left|R_{n}(x)\right| d x \leq & \int_{0}^{\pi}\left|\sum_{p=0}^{n} S_{p}^{k-\delta}(x) A_{n+1-p}^{\delta-1}\right| d x\left|\Delta^{k+1} a_{n+1}\right| \\
& +\int_{0}^{\pi}\left|\sum_{p=0}^{n} S_{p}^{k-\delta}(x) A_{n+2-p}^{\delta-1}\right| d x\left|\Delta^{k+1} a_{n+2}\right|+\cdots
\end{aligned}
$$

By (3.1) of the proof of Lemma 4

$$
\left|\sum_{p=0}^{n} S_{p}^{k-\delta}(x) A_{m-p}^{\delta-1}\right| \leq \sum_{p=0}^{n}\left|S_{p}^{k}(x)\right| \sum_{\mu=0}^{n-p} A_{\mu}^{-\delta-1} A_{m-p-\mu}^{\delta-1}
$$

for $m=n+1, n+2, \ldots$ Therefore

$$
\begin{aligned}
\int_{0}^{\pi}\left|R_{n}(x)\right| d x & \leq \int_{0}^{\pi} \sum_{p=0}^{n}\left|S_{p}^{k}(x)\right| \sum_{\mu=0}^{n-p} A_{\mu}^{-\delta-1} A_{n+1-p-\mu}^{\delta-1}\left|\Delta^{k+1} a_{n+1}\right| d x+\cdots \\
& =\int_{0}^{\pi} \sum_{p=0}^{n}\left|T_{p}^{k}(x)\right| A_{p}^{k} \sum_{\mu=0}^{n-p} A_{\mu}^{-\delta-1} A_{n+1-p-\mu}^{\delta-1}\left|\Delta^{k+1} a_{n+1}\right| d x+\cdots \\
& \leq \sum_{p=0}^{n} \int_{0}^{\pi}\left|T_{p}^{k}(x)\right| A_{p}^{k} \sum_{\mu=0}^{n-p} A_{\mu}^{-\delta-1} A_{n+1-p-\mu}^{\delta-1}\left|\Delta^{k+1} a_{n+1}\right| d x+\cdots \\
& \leq C \sum_{p=0}^{n} A_{p}^{k} \sum_{\mu=0}^{n-p} A_{\mu}^{-\delta-1} A_{n+1-p-\mu}^{\delta-1}\left|\Delta^{k+1} a_{n+1}\right|+\cdots
\end{aligned}
$$

by Lemma 3,

$$
=C \sum_{p=0}^{n} A_{p}^{k-\delta} A_{n+1-p}^{\delta-1}\left|\Delta^{k+1} a_{n+1}\right|+C \sum_{p=0}^{n} A_{p}^{k-\delta} A_{n+2-p}^{\delta-1}\left|\Delta^{k+1} a_{n+2}\right|+\cdots
$$

by applying the Abel transformation of order $\delta$,

$$
\begin{aligned}
& \leq C \sum_{p=0}^{n+1} A_{p}^{k-\delta} A_{n+1-p}^{\delta-1}\left|\Delta^{k+1} a_{n+1}\right|+C \sum_{p=0}^{n+2} A_{p}^{k-\delta} A_{n+2-p}^{\delta-1}\left|\Delta^{k+1} a_{n+2}\right|+\cdots \\
& =C A_{n+1}^{k}\left|\Delta^{k+1} a_{n+1}\right|+C A_{n+2}^{k}\left|\Delta^{k+1} a_{n+2}\right|+\cdots
\end{aligned}
$$

by (2.4). Thus by condition (b) of our theorem

$$
\int_{0}^{\pi}\left|R_{n}(x)\right| d x=o(1) \quad \text { as } n \rightarrow \infty
$$

and hence by (4.4)

$$
\int_{0}^{\pi}\left|h(x)-h_{n}(x)\right| d x=o(1) \quad \text { as } n \rightarrow \infty
$$

This implies that

$$
h_{n} \rightarrow h \text { in the } L^{1} \text {-metric. }
$$


Case (3). Let $k=r$. In this case

$$
h_{n}(x)=\sum_{p=0}^{n} S_{p}^{k-1}(x) \Delta^{k} a_{p} .
$$

Applying the Abel transformation, we have

$$
h_{n}(x)=\sum_{p=0}^{n} S_{p}^{k}(x) \Delta^{k+1} a_{p}+S_{n}^{k}(x) \Delta^{k} a_{n+1} .
$$

Since $S_{n}^{k}(x)$ is bounded for $0<\delta \leq x \leq \pi$ and $\Delta^{k} a_{n+1} \rightarrow 0$ as $n \rightarrow \infty$, so

$$
\lim _{n \rightarrow \infty} S_{n}^{k}(x) \Delta^{k} a_{n+1}=0 \text { for } 0<\delta \leq x \leq \pi \text {. }
$$

Hence

$$
h_{n}(x) \rightarrow h(x)=\sum_{p=0}^{\infty} S_{p}^{k}(x) \Delta^{k+1} a_{p}
$$

pointwise for $0<\delta \leq x \leq \pi$. Lastly,

$$
\begin{aligned}
\int_{0}^{\pi} \mid & h(x)-h_{n}(x)\left|d x=\int_{0}^{\pi}\right| \sum_{p=n+1}^{\infty} S_{p}^{k}(x) \Delta^{k+1} a_{p}-S_{n}^{k}(x) \Delta^{k} a_{n+1} \mid d x \\
\leq & \sum_{p=n+1}^{\infty} \int_{0}^{\pi}\left|S_{p}^{k}-(x)\right| d x\left|\Delta^{k+1} a_{p}\right|+\int_{0}^{\pi}\left|S_{n}^{k}(x)\right| d x\left|\Delta^{k} a_{n+1}\right| \\
= & \sum_{p=n+1}^{\infty} A_{p}^{k}\left|\Delta^{k+1} a_{p}\right| \int_{0}^{\pi}\left|T_{p}^{k}(x)\right| d x \\
& +A_{n+1}^{k}\left|\Delta^{k} a_{n+1}\right| \int_{0}^{\pi}\left|T_{n}^{k}(x)\right| d x \\
\leq & C \sum_{p=n+1}^{\infty} A_{p}^{k}\left|\Delta^{k+1} a_{p}\right|+C A_{n+1}^{k}\left|\Delta^{k} a_{n+1}\right|, \quad \text { by Lemma 3 } \\
= & o(1)+o(1) \\
= & o(1)
\end{aligned}
$$

by the hypothesis of the theorem and Lemma 1 . Thus $h_{n} \rightarrow h$ in the $L^{1}$-metric.

This complete the proof of the theorem.

\section{REFERENCES}

1. A. N. Kolmogorov, Sur l'ordre de grandeur des coefficients de la série de Fourier-Lebesgue, Bull. Acad. Polon. Sci. Ser. Sci. Math. Astronom. Phys. (1923), 83-86.

2. C. N. Moore, On the use of Cesàro means in determining criteria for Fourier constants, Presented to the Society and Section A of the American Association for the Advancement of Science, by invitation, June 21, 1933, on the occasion of an address by Professor L. Fejér.

3. A. Zygmund, Trigonometric series, Volume 1, Cambridge at the University Press, 1959.

4. L. S. Bosanquet, Note on convergence and summability factors. III, Proc. London Math. Soc. (2) 50 (1949), 482-495.

5. A. F. Andersen, On extensions within the theory of Cesàro summability of a classical convergence theorem of Dedekind, Proc. London Math. Soc. (3) 8 (1958), 1-52. 
6. C. S. Rees and C. V. Stanojević, Necessary and sufficient conditions for integrability of certain cosine sums, J. Math. Anal. Appl. 43 (1973), 579-586.

7. J. W. Garrett and C. V. Stanojevic, On integrability and $L^{1}$-convergence of certain cosine sums, Notices Amer. Math. Soc. 22 (1975), A-166.

8. Math. Soc. 60 (1976), 68-71.

9. Niranjan Singh and K. M. Sharma, $L^{1}$-convergence of modified cosine sums with generalized quasiconvex coefficients, J. Math. Anal. Appl. (to appear).

Department of MAThematics, KURUKShetra UNIVERSity, KURUKShetra-132 119 (HARYANA), INDIA 